\title{
The Accuracy of Imminent Death Diagnosis in Palliative Care setting
}

\author{
Abdullah Alsuhail MD1, Balaji Duraisamy MD1 , Abdullah Rehaili MD1, Sami Ayed Alshammary MD1,2
}

1 King Fahad Medical City, Riyadh, Saudi Arabia.

2 Centre for Postgraduate Studies in Family Medicine, Riyadh, Saudi Arabia

\section{Introduction}

Prognostication or estimation of time of death is important for the patients and their family members as they need this information for preparation and planning their future and for the physicians as they desire to be accurate in their prognostication skills in order to plan and deliver better care.

\section{Objectives}

The study aims to evaluate the current situation regarding the accuracy of palliative care physicians' clinical predictions of time of death from initiation of imminent death protocol. The research hypothesis suggests that patients will die within 14 days' post imminent death diagnosis (IDD).

\section{Methods}

The imminent death protocol was developed for very sick patients who were expected to survive for less than two weeks. In order to initiate the imminent death protocol, the patient has to be assessed by two palliative care physicians who can diagnose a patient for imminent death. The time between imminent death diagnosis (IDD) and death will be documented.

\section{Results}

During study period, 48 patients died in PCU. The majority 28/48 (58\%) died with imminent death diagnosis (IDD). In IDD group, 25/28(89.3) died within 14 days of diagnosis while $3 / 28$ (10.3\%) died after 14 days. The median survival is 50 hours (Cl 35-85).

\section{Conclusions}

Imminent death diagnosis (IDD) is a critical skill for Palliative care physicians to make a proper care plan. Our study showed that $90 \%$ of PCU patient died within 14 days' post imminent death diagnosis. The median survival was two days. We recommend further study to explore how IDD could improve care plan for dying patients. 